\title{
Hierarchical linear modeling of longitudinal pedigree data for genetic association analysis
}

\author{
Qihua Tan ${ }^{1,2^{*}}$, Jacob V B Hjelmborg ${ }^{2}$, Mads Thomassen ${ }^{1}$, Andreas Kryger Jensen², Lene Christiansen ${ }^{1,2}$, \\ Kaare Christensen ${ }^{1,2}$, Jing Hua Zhao ${ }^{3}$, Torben A Kruse ${ }^{1}$ \\ From Genetic Analysis Workshop 18 \\ Stevenson, WA, USA. 13-17 October 2012
}

\begin{abstract}
Genetic association analysis on complex phenotypes under a longitudinal design involving pedigrees encounters the problem of correlation within pedigrees, which could affect statistical assessment of the genetic effects. Approaches have been proposed to integrate kinship correlation into the mixed-effect models to explicitly model the genetic relationship. These have proved to be an efficient way of dealing with sample clustering in pedigree data. Although current algorithms implemented in popular statistical packages are useful for adjusting relatedness in the mixed modeling of genetic effects on the mean level of a phenotype, they are not sufficiently straightforward to handle the kinship correlation on the time-dependent trajectories of a phenotype. We introduce a 2-level hierarchical linear model to separately assess the genetic associations with the mean level and the rate of change of a phenotype, integrating kinship correlation in the analysis. We apply our method to the Genetic Analysis Workshop 18 genome-wide association studies data on chromosome 3 to estimate the genetic effects on systolic blood pressure measured over time in large pedigrees. Our method identifies genetic variants associated with blood pressure with estimated inflation factors of 0.99 , suggesting that our modeling of random effects efficiently handles the genetic relatedness in pedigrees. Application to simulated data captures important variants specified in the simulation. Our results show that the method is useful for genetic association studies in related samples using longitudinal design.
\end{abstract}

\section{Background}

Genetic studies show that genes influence both the mean level and the rate of change of anthropometric traits. For example, substantial genetic contributions to the rate of change have been detected for both body mass index (BMI) [1,2] and body weight [3] using longitudinal data on twins. These results provide an epidemiological basis for molecular genetic studies to identify genetic variants that affect these traits. Genome-wide association studies (GWAS) driven by high-throughput genotyping techniques enable the mapping of genes associated with changes in human traits using the longitudinal design, which has been shown to have power advantages over the crosssectional design $[4,5]$. For example, in a longitudinal

\footnotetext{
* Correspondence: qtan@health.sdu.dk

'Institute of Clinical Research, Unit of Human Genetics, University of

Southern Denmark, Sdr. Boulevard 29, 5000 Odense C, Denmark

Full list of author information is available at the end of the article
}

GWAS conducted on cardiovascular disease (CVD) risk factors, Smith et al [6] detected single-nucleotide polymorphisms (SNPs) that influence the change in multiple traits or CVD risk factors including glucose, low-density lipoprotein, triglyceride levels, body weight, and waist circumference, among others. Another very recent longitudinal GWAS on BMI identified SNPs associated with development of obesity [7].

Just as traditional genetic epidemiology studies are frequently conducted in large pedigrees because diseasecausing mutations segregate within families, so the relative ease in SNP genotyping has led to genetic association analysis in large pedigrees. For example, the Long Life Family Study (https://dsgweb.wustl.edu/llfs/), supported by the National Institute on Aging, collects high-resolution genome-wide genotype data in families with longevity probands and their offspring. With support from the 
NIH, Genetic Analysis Workshop 18 (GAW18) provides whole genome sequencing data and longitudinal blood pressure measurements on large pedigrees. In fact, genetic analysis on longitudinal measurements from pedigrees is an important topic calling for novel statistical modeling $[8,9]$. Such data are characterized by the random variation between individual longitudinal trajectories arising from repeated measurement on an individual, and by the random effect between pedigrees resulting from relatedness among individuals within a pedigree, which is one of the situations of sample stratification in GWAS [10]. An efficient mixed model was introduced to deal with sample structure resulting from genetic correlation by introducing a kinship matrix to account for pairwise relatedness between individual samples [11]. Implementation of the algorithm to GWAS in correlated samples is possible via software packages such as EMMAX [11] and kinship [12]. Although useful for adjusting relatedness in the mixed modeling of genetic effects on the mean level of a phenotype, current algorithms implemented in popular statistical packages are not sufficiently straightforward for handling the kinship correlation on the time-dependent trajectories of a phenotype. This paper introduces a novel integration of the hierarchical linear model (HLM) to handle intraindividual correlation resulting from repeated longitudinal measurements and the kinship model to deal with intrapedigree relatedness with an example application to the GAW18 data.

\section{Methods \\ The GAW18 data \\ Phenotype data}

GAW18 provides blood pressure data in 20 large pedigrees (27 to 107 individuals per pedigree, mean pedigree size 69 individuals, 932 participants in total) measured longitudinally over 4 times in a period of 30 years. In total, 246 individuals have 1, 183 have 2, 309 have 3, and 194 have 4 measurements. Besides blood pressure, information concerning hypertension diagnosis (systolic blood pressure $[\mathrm{SBP}]>140 \mathrm{~mm} \mathrm{Hg}$, diastolic blood pressure $[\mathrm{DBP}]>90$ $\mathrm{mm} \mathrm{Hg}$ ), antihypertension medicine intake, and tobacco smoking is also collected at each examination. Participants entered the study at different ages, ranging from 16 to 94 years, with a mean age of 39.6 years.

\section{Genotype data}

The data contains SNP genotypes on odd-numbered autosomes for individuals in the 20 pedigrees obtained using different versions of the Illumina Infinium Beadchips. Raw genotype data were processed using standard quality control procedures and cleaned for mendelian errors. This paper focuses on chromosome 3 data, which contains 65,519 SNPs, to illustrate the application of our proposed method.

\section{Simulated data}

GAW18 also provides simulated phenotype data for 200 replicates, each with 849 individuals from the real pedigrees. As with the real data, longitudinal blood pressure measurements were simulated for 3 time points with 3.9 years between exams 1 and 2 and 6.9 years between exams 1 and 3. The simulation included age and sex as fixed covariates and hypertension diagnosis, medication use, and tobacco smoking as time-varying covariates.

\section{Hierarchical linear models}

The HLM is a complex form of regression analysis $[13,14]$, referred to as random coefficients model with 2 defining features. First, the data appropriate for HLM are structured with difference levels with lower-level or level 1 units (here, blood pressure measurements over time for each individual) nested within the higher-level or level 2 units (here, genotypes at a given SNP locus across individuals). Second, the parameters of the level 1 model characterize linear relationships occurring between level 1 units (here, the blood pressure trajectory over time). These parameters can be modeled as a function of level 2 units (genotypes). Our 2-level HLM takes the form of regression models developed separately for level 1 and level 2 units. For clarity, the level 1 model for each individual can be shown as

$$
Y_{i j}=\beta_{0, i}+\beta_{1, i} X_{i j}+e_{i j}
$$

where $Y_{i j}$ is SBP measured for individual $i$ at age $j ; X_{i j}$ is age at measurement for individual $i ; \beta_{0, i}$ and $\beta_{1, i}$ are the intercept and slope parameters for individual $i$; $e_{i j}$ is a random error associated with individual $i$ at age $j$ that is normally distributed with $E\left(e_{i j}\right)=0, \operatorname{var}\left(e_{i j}\right)=\sigma^{2}$.

In the level 2 model, the regression coefficients from the level 1 model are regressed on the level 2 group variable, here defined as the genotypes for a SNP:

$$
\begin{aligned}
& \beta_{0, i}=\gamma_{00}+\gamma_{01} G_{i}+U_{0, i} \\
& \beta_{1, i}=\gamma_{10}+\gamma_{11} G_{i}+U_{1, i}
\end{aligned}
$$

In equations (2) and (3), $G_{i}$ is genotype for individual $i$; $\gamma_{0 o}$ and $\gamma_{10}$ are the intercepts or overall means for $\beta_{0, i}$ and $\beta_{1, i} ; \gamma_{01}$ and $\gamma_{11}$ are the regression coefficients (slopes) associating genotypes with $\beta_{0, i}$ and $\beta_{1, i} ; U_{0 i}$ and $U_{1 i}$ are the random effects for $\beta_{0, i}$ and $\beta_{1, i}$ after adjusting for genotype $G_{i}$ that are normally distributed with $E\left(U_{O i}\right)=E$ $\left(U_{1 i}\right)=0$. In our context, the most interesting parameters are $\gamma_{01}$ and $\gamma_{11}$, which represent genotype association with overall mean and rate of change for SBP.

\section{Modeling relatedness}

The HLMs described so far are most appropriate for unrelated individuals from a general population. When 
longitudinal data are collected in pedigrees, correlation arises among the random effects for individual trajectories described above. In human pedigrees, the heritable random variation is distributed following a kinship matrix defined according to the pairwise genotypic similarity of individuals, which can be readily derived from pedigree structures or whole genome data. Explicitly modeling the pedigree structure in a mixed-effect model is shown to improve performance in GWAS by bringing the genomic control factor very close to $1[11,15]$. Here we introduce a mixed model that involves a kinship matrix to our level 2 models to handle the relatedness resulting from pedigree structures in the GAW18 data. In the mixed model, we define $G_{i}$ in equations (2) and (3) as a fixed-effect variable and $U_{0, i}$ and $U_{1, i}$ as random effect variables that can be decomposed into 2 parts: a heritable component $\left(u_{0, i}\right.$ or $\left.u_{1 i}\right)$ and a nonheritable component $\left(\varepsilon_{0, i}\right.$ or $\left.\varepsilon_{1, i}\right)$ such that

$$
\begin{aligned}
& U_{0, i}=u_{0, i}+\varepsilon_{0, i} \\
& U_{1, i}=u_{1, i}+\varepsilon_{1, i} \\
& \operatorname{Var}\left(u_{0}\right)=\sigma_{0,8}^{2} K, \operatorname{Var}\left(u_{1}\right)=\sigma_{1,8}^{2} K
\end{aligned}
$$

In equation (6), $u_{0}$ is a vector representing the heritable component in the random variation in individual intercepts, $\beta_{0, i} ; u_{1}$ is a vector representing the heritable component in the random variation in individual slopes, $\beta_{1, i} ; \sigma_{0, g}^{2}$ and $\sigma_{1, g}^{2}$ represent the variability in phenotype dissimilarity among genetically related individuals; $K$ is a matrix of kinship coefficients calculated from pedigree structures. In the actual estimation of the level 2 models, extra fixed-effect variables, such as sex, can be included so that effects of these fixed variables can be estimated together with that for each SNP with the possibility of estimating interactions between them. The level 2 models are fitted using the lmekin function in the free $\mathrm{R}$ package, kinship.

\section{Results}

\section{Original phenotype data}

We started with regressing SBP values on 2 time-varying variables, smoking and intake of antihypertension medicine, to remove their effects, and then fitted the level 1 models to the age trajectories of SBP measured over time. Figure 1 is a histogram for the estimated SBP at age 42 years (the mean age for all ages at examination), or SBP (42), and the slope for each individual. There are outliers at the far ends in the distributions (Figure 1), defined as

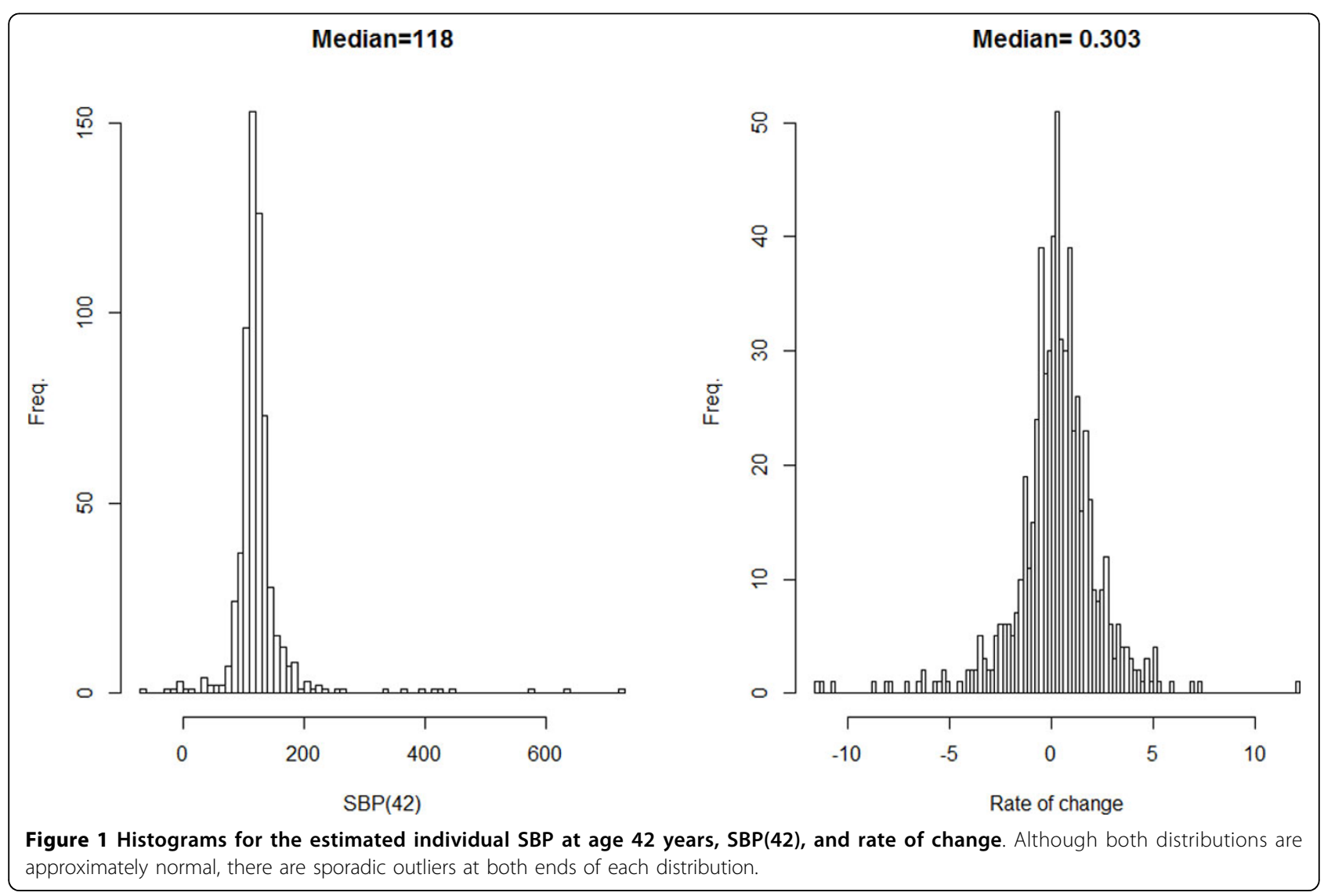


those whose values are beyond 3 standard deviations from the mean, that were removed in fitting the level 2 models. The median for SBP(42) is $118 \mathrm{~mm} \mathrm{Hg}$. The median for the slope is $0.303 \mathrm{~mm} \mathrm{Hg}$ per year, which means that for every 10-year increase in age, SBP is expected to increase about $3 \mathrm{~mm} \mathrm{Hg}$ on average.

For each of the 65,519 SNPs genotyped on chromosome 3, we next fitted the level 2 models as described in equations (2) to (6) and included sex as an additional fixed-effect variable. The Q-Q plot is shown in Figure 2 for the $p$ values for effects of each SNP on SBP(42) (the left panel) and on rate of change (the right panel). Although no SNP reached genome significance ( $p$ value $<5 \mathrm{e}-08$ ), some SNPs on chromosome 3 may tend to affect the mean level of SBP (SNP IDs are shown in Figure 2). Meanwhile, our analysis did not reveal any genetic associations that affect the rate of change in SBP for the tested SNPs on chromosome 3. The estimated inflation factors $(\lambda)$ are 0.99 for both SBP(42) and rate of change, suggesting that our modeling of random effects efficiently removed relatedness in substructures resulting from repeated measurements within individuals and between individuals within pedigrees.

\section{Simulated phenotype data}

The application to original data has shown that the influence of pedigree structure on statistical testing can be nicely controlled by our method. We next use the simulated phenotype data to validate performance of our model and to see whether important genetic variants can be captured for given sample size in the simulation. As with the real data, we focused only on SBP and used regression models to adjust for effects of time-varying variables (smoking and medication) and fitted level 1 model to the residuals. Considering the enormous computational load in performing GWAS for 200 replicates, we limited our analysis on simulated data to all SNPs in the MAP4 gene on chromosome 3. The simulation included 14 functional variants (6 with minor allele frequency $[\mathrm{MAF}]>0.01$ ) accounting for $7.7 \%$ of the total variance in SBP. A total of 87 SNPs in the GAW18 GWAS data were mapped to the MAP4 gene. We performed level 2 analysis on all 200 replicates, focusing on 62 SNPs with MAF $>0.01$. Based on the results on simulated replicates, statistical power was calculated as defining type I error rate $\alpha=0.05$ and $\alpha=$ $0.05 /$ number of SNPs tested ( 0.0008). With a sample size of 849 individuals, the highest power in detecting the effect on mean SBP or SBP(42) was achieved by SNP rs11711953 $(\mathrm{MAF}=0.028)$ with power estimates of 1 and 0.70 for $\alpha$ values of 0.05 and 0.0008 . This SNP is the variant in MAP4 assigned the highest effect on SBP, accounting for $2.78 \%$ of the variance in SBP. Interestingly, we also obtained the highest power for detecting association with rate of change from the same SNP (0.75 for $\alpha=0.05)$, suggesting that rs11711953 influences both the mean level (negative correlation) and the rate of change over ages (positive correlation) of the simulated SBP. Table 1 displays the top 10 SNPs showing the highest power. As can be seen, the statistical
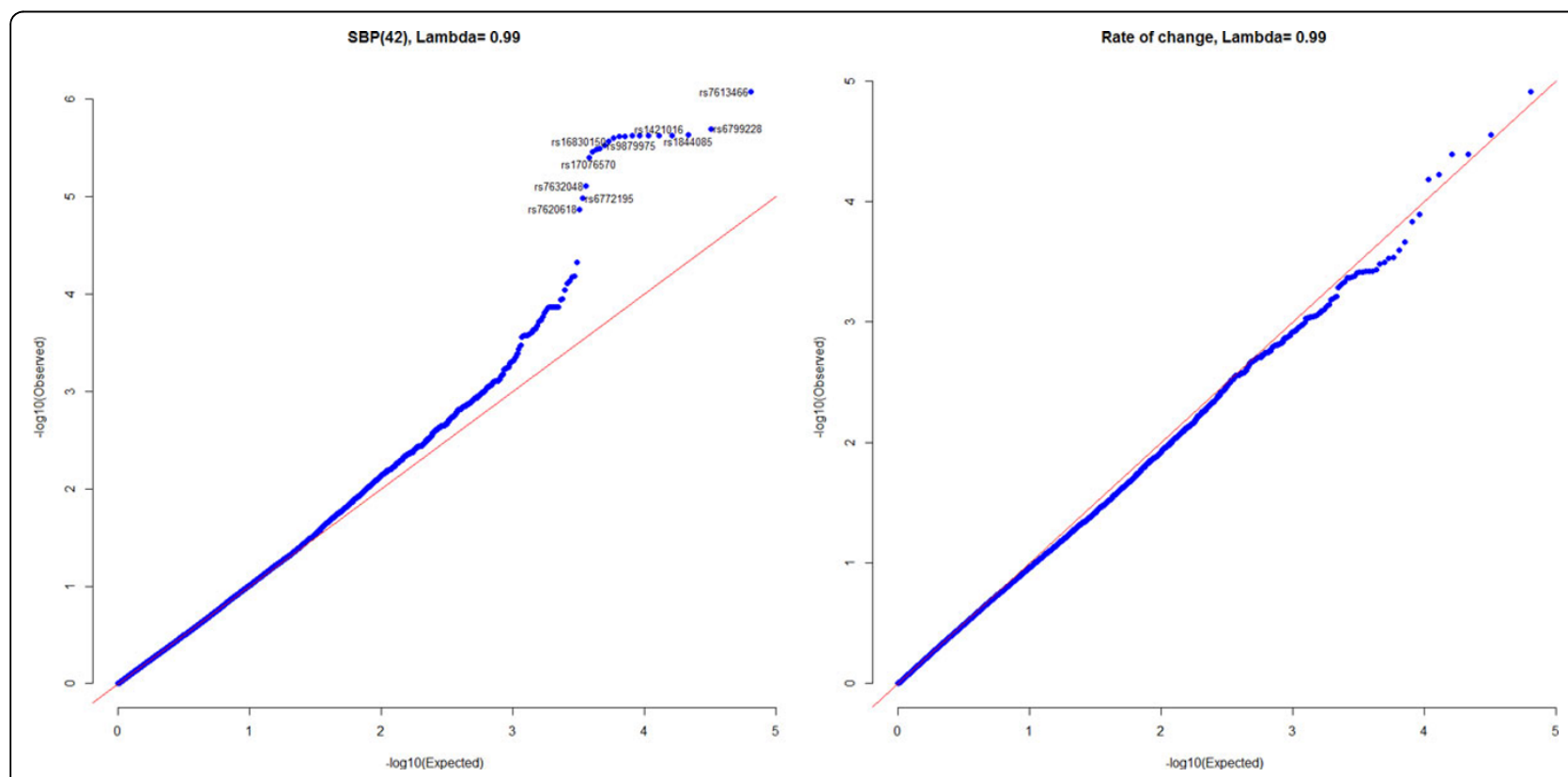

Figure 2 Q-Q plots for the observed against expected $p$ values in association analysis of chromosome 3 data, for SBP(42) (left panel) and for rate of change (right panel). 
Table 1 The top 10 SNPs (MAF >0.01) detected with the highest statistical power from simulated data

\begin{tabular}{|c|c|c|c|c|c|c|c|c|c|c|}
\hline \multirow[b]{2}{*}{ SNP } & \multirow[b]{2}{*}{ Position } & \multirow[b]{2}{*}{ MAF } & \multicolumn{2}{|c|}{ SBP(42) } & \multicolumn{2}{|c|}{ Rate of change } & \multicolumn{4}{|c|}{ Closest functional variants in simulation } \\
\hline & & & Power $\alpha=0.05$ & Beta mean & Power $\alpha=0.05$ & Beta mean & Position & MAF & Beta & $\%$ Variance \\
\hline rs11711953 & 48040283 & 0.03 & 1.00 & -18.6 & 0.75 & 0.59 & $\begin{array}{l}48042083 \\
48042084\end{array}$ & $\begin{array}{l}0.03 \\
0.01\end{array}$ & $\begin{array}{l}-9.91 \\
-11.01\end{array}$ & $\begin{array}{l}0.028 \\
0.011\end{array}$ \\
\hline rs1665982 & 47905079 & 0.11 & 0.64 & -6.08 & 0.46 & 0.25 & $\begin{array}{l}48042083 \\
48042084 \\
47913455\end{array}$ & $\begin{array}{l}0.03 \\
0.01 \\
0.005\end{array}$ & $\begin{array}{l}-9.91 \\
-11.01 \\
-8.70\end{array}$ & $\begin{array}{l}0.028 \\
0.011 \\
0.004\end{array}$ \\
\hline rs319680 & 47898307 & 0.15 & 0.53 & -4.60 & 0.41 & 0.20 & 47913455 & 0.005 & -8.70 & 0.004 \\
\hline rs6763824 & 47905427 & 0.14 & 0.46 & -4.47 & 0.38 & 0.20 & 47913455 & 0.005 & -8.70 & 0.004 \\
\hline rs184388 & 47939626 & 0.34 & 0.40 & -3.03 & 0.33 & 0.13 & 47956424 & 0.38 & -2.38 & 0.014 \\
\hline rs1060407 & 47958037 & 0.34 & 0.39 & -3.02 & 0.33 & 0.13 & $\begin{array}{l}47957996 \\
47957741\end{array}$ & $\begin{array}{l}0.03 \\
0.002\end{array}$ & $\begin{array}{l}-7.39 \\
-8.10\end{array}$ & $\begin{array}{l}0.015 \\
0.003\end{array}$ \\
\hline rs7430879 & 48038714 & 0.38 & 0.39 & -2.82 & 0.39 & 0.13 & $\begin{array}{l}48042083 \\
48042084\end{array}$ & $\begin{array}{l}0.03 \\
0.01\end{array}$ & $\begin{array}{l}-9.91 \\
-11.01\end{array}$ & $\begin{array}{l}0.028 \\
0.011\end{array}$ \\
\hline rs4599334 & 48066400 & 0.35 & 0.37 & -2.93 & 0.29 & 0.12 & $\begin{array}{l}48042083 \\
48042084\end{array}$ & $\begin{array}{l}0.03 \\
0.01\end{array}$ & $\begin{array}{l}-9.91 \\
-11.01\end{array}$ & $\begin{array}{l}0.028 \\
0.011\end{array}$ \\
\hline rs4296617 & 48068308 & 0.35 & 0.37 & -2.92 & 0.28 & 0.12 & $\begin{array}{l}48042083 \\
48042084\end{array}$ & $\begin{array}{l}0.03 \\
0.01\end{array}$ & $\begin{array}{l}-9.91 \\
-11.01\end{array}$ & $\begin{array}{l}0.028 \\
0.011\end{array}$ \\
\hline rs2053767 & 47999674 & 0.38 & 0.37 & -2.75 & 0.38 & 0.13 & $\begin{array}{l}48042083 \\
48042084\end{array}$ & $\begin{array}{l}0.03 \\
0.01\end{array}$ & $\begin{array}{l}-9.91 \\
-11.01\end{array}$ & $\begin{array}{l}0.028 \\
0.011\end{array}$ \\
\hline
\end{tabular}

power for each of the SNPs depends on the effect and frequency of the genetic variants in their vicinity and the distance to them. Interestingly, the functional variants in the close vicinity of the top 6 SNPs explain $7.5 \%$ of the total variance in SBP, which is nearly the total effect of the MAP4 gene specified in the simulation.

\section{Discussion}

In longitudinal investigations, researchers are interested in studying interindividual differences in intraindividual changes. Such studies are inherently hierarchical in nature. The nested structure in data includes multiple or repeated observations within an individual, and a collection of multiple individuals in a sample, which can be modeled by HLM. The nested modeling is actually equivalent to the mixed-effects model from a theoretical prospective. By incorporating equations (2) and (3) into equation (1), we obtain

$$
Y_{i, j}=\gamma_{00}+\gamma_{10} X_{i j}+\gamma_{01} G_{i}+\gamma_{11} G_{i} X_{i j}+U_{1, i} X_{i j}+U_{0, i}+r_{i j}(7)
$$

The combined model has fixed effects for age, genotype and their interaction, and the composite error including random effects for the age trajectory and for the mean SBP level. Unlike the mixed model that corrects phenotype correlation within pedigree, our modeling strategy allows a direct adjustment of kinship correlation in the mean level and the rate of change of SBP. In a combined model, however, this is somewhat more difficult to implement. As shown by the application example, our hierarchical modeling strategy enables integration of kinship matrix to explicitly account for the genetic correlation both in the level and in the rate of change of SBP in general pedigrees using readily available software packages. For comparison, we performed similar association analysis on chromosome 3 but using the linear mixed model with individual rate of change as dependent variable and assuming random effect for each pedigree. The estimated inflation factor becomes 1.07, indicating a moderate degree of sample substructure. This is in contrast with a lambda of 1 from the lmekin model, suggesting that explicitly modeling the kinship correlation structure can help to improve model performances for GWAS in pedigree studies.

Our hierarchical modeling procedure also offers an easy way to detect outliers in longitudinal studies [16], which is easily done by examining the distribution of the estimated individual intercepts and slopes (see Figure 1). For example, in our analysis, we define outliers as any observation with values for the intercept or $\operatorname{SBP}(42)$ and for the slope or rate of change beyond 3 standard deviations from the mean. In addition, we also set those with an estimated $\mathrm{SBP}(42)$ of less than $60 \mathrm{~mm} \mathrm{Hg}$ as outliers because it is unreasonable for SBP to be too low to maintain physical function (normal range for SBP: 90 to $140 \mathrm{~mm} \mathrm{Hg}$ ). Because the individual intercept and slope are based on the level 1 model fitted to the repeated measurements on each participant, our multilevel modeling process actually provides summary metrics that can be used for detecting outliers. Note that, in longitudinal studies, the increasing number of waves for data collection can complicate the search for outliers. However, this is an advantage for our way of defining outliers because, with repeated observations, the resulting random slope for each individual can be more stabilized and definition of outliers less affected by occasional extreme point observations. 
Finally, although only chromosome 3 data are used as an example, application of the method to genome-wide data is straightforward. The fast fitting of the mixed model with a predefined kinship matrix enables computational feasibility for large GWAS data. With the increasing popularity of GWAS, we hope that the proposed method can be helpful in analyzing data of related individuals using both cross-sectional and longitudinal designs.

\section{Conclusion}

Hierarchical linear modeling of longitudinal pedigree data can handle relatedness in detecting genetic variations that affect the mean level or the rate of change for a phenotype of interest in genetic association analysis.

\section{Competing interests}

The authors declare that they have no competing interests.

\section{Authors' contributions}

TAK, KC, and JBH designed the overall study; QT, AKJ, and JZ conducted statistical analyses. QT, MT, and LC drafted the manuscript. All authors read and approved the final manuscript.

\section{Acknowledgements}

The GAW18 whole genome sequence data were provided by the T2DGENES Consortium, which is supported by NIH grants U01 DK085524, U01 DK085584, U01 DK085501, U01 DK085526, and U01 DK085545. The other genetic and phenotypic data for GAW18 were provided by the San Antonio Family Heart Study and San Antonio Family Diabetes/Gallbladder Study, which are supported by NIH grants P01 HL045222, R01 DK047482, and R01 DK053889. The Genetic Analysis Workshop is supported by NIH grant R01 GM031575. This work was also partially supported by the Long Life Family Study (LLFS) under the National Institute on Aging (NIA).

\section{Declarations}

This article has been published as part of BMC Proceedings Volume 8 Supplement 1, 2014: Genetic Analysis Workshop 18. The full contents of the supplement are available online at http://www.biomedcentral.com/bmcproc/ supplements/8/S1. Publication charges for this supplement were funded by the Texas Biomedical Research Institute.

\section{Authors' details}

'Institute of Clinical Research, Unit of Human Genetics, University of Southern Denmark, Sdr. Boulevard 29, 5000 Odense C, Denmark.

'Epidemiology, Biostatistics and Biodemography, Institute of Public Health, University of Southern Denmark, J. B. Winsloews Vej 9B, 5000 Odense C, Denmark. ${ }^{3} \mathrm{MRC}$ Epidemiology Unit and Institute of Metabolic Science, Addenbrooke's Hospital, Cambridge CB2 0QQ, UK.

Published: 17 June 2014

\section{References}

1. Austin MA, Friedlander Y, Newman B, Edwards K, Mayer-Davis EJ, King MC: Genetic influences on changes in body mass index: a longitudinal analysis of women twins. Obes Res 1997, 5:326-331.

2. Hjelmborg JB, Fagnani C, Silventoinen $K$, McGue M, Korkeila M, Christensen K, Rissanen A, Kaprio J: Genetic influences on growth traits of BMI: a longitudinal study of adult twins. Obesity (Silver Spring) 2008, 16:847-852.

3. Fabsitz RR, Sholinsky P, Carmelli D: Genetic influences on adult weight gain and maximum body mass index in male twins. Am J Epidemiol 1994, $140: 711-720$
4. Macgregor S, Knott SA, White I, Visscher PM: Quantitative trait locus analysis of longitudinal quantitative trait data in complex pedigrees. Genetics 2005, 171:1365-1376.

5. Shi G, Rao DC: Ignoring temporal trends in genetic effects substantially reduces power of quantitative trait linkage analysis. Genet Epidemiol 2008, 32:61-72.

6. Smith EN, Chen W, Kähönen $M$, Kettunen J, Lehtimäki T, Peltonen L, Raitakari OT, Salem RM, Schork NJ, Shaw M, et al: Longitudinal genomewide association of cardiovascular disease risk factors in the Bogalusa Heart Study. PLOS Genet 6:e1001094.

7. Mei H, Chen W, Jiang F, He J, Srinivasan S, Smith EN, Schork N, Murray S, Berenson GS: Longitudinal replication studies of GWAS risk SNPs influencing body mass index over the course of childhood and adulthood. PLoS One 2012, 7:e31470.

8. Zhu W, Cho K, Chen X, Zhang M, Wang M, Zhang H: A genome-wide association analysis of Framingham Heart Study longitudinal data using multivariate adaptive splines. BMC Proc 2009, 3(Suppl 7):S119.

9. Wang $Y$, Huang $C$, Fang $Y$, Yang $Q$, Li R: Flexible semiparametric analysis of longitudinal genetic studies by reduced rank smoothing. J $R$ Stat SOC Ser C Appl Stat 2012, 61:1-24.

10. Price $A L$, Zaitlen NA, Reich $D$, Patterson N: New approaches to population stratification in genome-wide association studies. Nat Rev Genet 2010, 11:459-463.

11. Kang HM, Sul JH, Service SK, Zaitlen NA, Kong SY, Freimer NB, Sabatti C, Eskin E: Variance component model to account for sample structure in genome-wide association studies. Nat Genet 2010, 42:348-354.

12. Zhao JH: Mixed-effects Cox models of alcohol dependence in extended families. BMC Genet 2005, 6(Suppl 1):S127.

13. Hofmann DA: An overview of the logic and rationale of hierarchical linear models. J Manage 1997, 23:723-744.

14. Woltman H, Feldstain A, MacKay JC, Rocchi M: An introduction to hierarchical linear modeling. Tutor Quant Methods Psychol 2012, 8:52-69.

15. Zhang Z, Ersoz E, Lai CQ, Todhunter RJ, Tiwari HK, Gore MA, Bradbury PJ, Yu J, Arnett DK, Ordovas JM, et al: Mixed linear model approach adapted for genome-wide association studies. Nat Genet 2010, 42:355-360.

16. Ecob R, Der G: An iterative method for the detection of outliers in longitudinal growth data using multilevel models. In Multilevel Modeling: Methodological Advances, Issues and Applications. Mahwah, New Jersey Lawrence Erlbaum;Reise SP, Duan N 2003:229-254.

doi:10.1186/1753-6561-8-S1-S82

Cite this article as: Tan et al:: Hierarchical linear modeling of longitudinal pedigree data for genetic association analysis. BMC Proceedings 2014 8(Suppl 1):S82.

\section{Submit your next manuscript to BioMed Central and take full advantage of:}

- Convenient online submission

- Thorough peer review

- No space constraints or color figure charges

- Immediate publication on acceptance

- Inclusion in PubMed, CAS, Scopus and Google Scholar

- Research which is freely available for redistribution

Submit your manuscript at www.biomedcentral.com/submit 